\title{
Characterization of neuronal apoptosis in the ventral ganglia of postembryonic silkworms Bombyx mori (Lepidoptera: Bombycidae)
}

\author{
Hwa Young SONG ${ }^{1 *}$, Chang Ok CHOI ${ }^{1 *}$, Mi Young KIM ${ }^{1}$, Bo Yong KIM ${ }^{1}$, Tae Joong YOON ${ }^{2}$, Dae Yong SONG 3 , \\ Jung Cheol PARK ${ }^{4}$, Pil Don KANG ${ }^{5}$, SAng Ho LEE ${ }^{1}$, Yeon JAe BAE ${ }^{1}$ and Bong Hee LEE ${ }^{1,2 * *}$ \\ ${ }^{1}$ School of Life Sciences and Biotechnology, Korea University, Seoul 136-701, Korea \\ ${ }^{2}$ Korean Entomological Institute, Korea University, Seoul 136-701, Korea \\ ${ }^{3}$ Department of Anatomy and Neurosciences, Eulji University, Daejeon 301-832, Korea \\ ${ }^{4}$ School of Natural Science, Baekseok Culture University, Cheonan 330-704, Korea \\ ${ }^{5}$ Department of Agricultural Biology, National Institute of Agricultural Science and Technology, Suwon 441-100, Korea
}

Key words. Neuronal apoptosis, fluorescent triple staining, ventral ganglia, Lepidoptera, Bombycidae, silkworm, 20E

\begin{abstract}
A fluorescent triple staining method was developed to stain the cytoplasm of neurons red, the nuclei of all kinds of cells, including neurons, blue and the nuclei of apoptotic neurons in cyan in the twelve ventral ganglia (VG) of the Bombyx mori ventral nerve cord. This differential staining method was used to distinguish between apoptotic and normal neurons in the suboesophageal ganglion (SOG), thoracic ganglia (TG)1 to TG3 and abdominal ganglia (AG)1 to AG8 and also determine the changes in the numbers of apoptotic neurons that occur during postembryonic development. In most of the VG tested, neuronal apoptosis was most marked during the period from the end of larval life to the mid pupal stage. The greatest number of apoptotic neurons was found in SOG of day-5 pupae, TG1 to TG3 and AG1 to AG4 of day-1 pupae, and AG5 to AG8 of day-4 pupae. In vivo injection of 20hydroxyecdysone (20E) into day-8 5 th instar larvae resulted in both a considerable increase in the number of apoptotic neurons and cleavage of procaspase-3 into caspase-3, which induced neuronal apoptosis in SOG and AG6 to AG8 in day-1 pupae, and a slight increase in the number of apoptotic neurons in TG1. In TG3 and AG4, however, it had little effect on the number of apoptotic neurons or cleavage of procaspase-3. Treatment of the VG of both day-8 5th instar larvae and day-2 pupae with protein synthesis inhibitors by in vivo injection triggered a significant inhibition of neuronal apoptosis and procaspase- 3 cleavage in most of these ganglia in day-1 pupae and day-4 pupae, but not TG3 and AG4, in which there was little procaspase-3 and caspase-3. In vivo injection of caspase- 8 and -3 inhibitors into day-8 5th instar larvae and day-2 pupae led to a substantial inhibition of neuronal apoptosis and of procaspase-3 cleavage in SOG, AG6 and TAG, but not in TG3 or AG4 of day-1 pupae and day-4 pupae. These findings suggest that neurons that die in SOG, TG1 and AG6 to AG8 in day-1 and -4 pupae may undergo apoptosis induced by the synthesis of a new protein and caspase-8- and -3-implicated signal transduction by the increase in titre of $20 \mathrm{E}$ in the haemolymph but not the neuronal aopotosis in TG3 and AG4. This study provides neurobiologists with valuable information and a means of studying neuronal apoptosis in the nervous system of insects.
\end{abstract}

\section{INTRODUCTION}

The central nervous system (CNS) of insects consists of the ventral nerve cord (VNC) and the brain (Bate \& Martinez-Arias, 1993). The larval VNC of the silkworm, $B$. mori, which is one of the most widely studied lepidopteran insects, is composed of one suboesophageal ganglion (SOG,) three thoracic ganglia (TG), and eight abdominal ganglia (AG) (Bullock \& Horridge, 1965). Immediately after holometabolous insects, such as the silkworm, metamorphose from larva to pupa, the larval VNC and associated segments gradually change into the adult form (Truman et al., 1993).

The metamorphic transformation of the larval into the adult VNC in insects is accompanied by a reduction in the length of the connections between ganglia and changes the number and structure of neurons within the ganglia (Truman et al., 1993; Niven et al., 2008). A reduction in the length of the connections between ganglia in $B$. mori leads to ganglionic fusion in the pupal stages: fusion of SOG into the brain, fusion of AG1 and AG2 into TG1 and TG2, and incorporation of AG6 into AG7/8 (Sato, 1998; Yamanaka et al., 2000; Hirashima et al., 2007; Mitsumasu et al., 2008). These ganglionic fusions are complete by day-4 of pupation. Most specific numerical changes in the neurons in the ventral ganglia (VG) of the $\mathrm{VNC}$, including programmed cell death (PCD), or apoptosis, occur during metamorphosis (Truman \& Schwartz, 1984).

Prior to the development by Loesel et al. (2006) of a simple fluorescent double staining method capable of distinguishing neuronal and non-neuronal cells in insect ganglia neuronal apoptosis was primarily studied by culturing specific neurons of certain Manduca sexta VG or the $B$. mori brain and treating them with 20-hydroxyecdysone (20E) (Levine \& Truman, 1985; Prugh et al., 1992; Strei-

\footnotetext{
* These authors should be considered equal first authors.

** Corresponding author; e-mail: bhlee@korea.ac.kr
} 
chert \& Weeks, 1995; Streichert et al., 1997; Hoffman \& Weeks, 1998; Zee \& Weeks, 2001; Park et al., 2003).

Horseradish peroxidase (HRP) is glycoprotein enzyme with a molecular weight of approximately $44 \mathrm{kDa}$. After combination with hydrogen peroxide $\left(\mathrm{HRP}-\mathrm{H}_{2} \mathrm{O}_{2}\right)$, this enzyme utilizes chemiluminescent substrates such as luminol and isoluminil in immunohistochemical reactions (Shannon et al., 1966; Welinder, 1978). Moreover, this protein is present in the cytoplasm of insect neurons. An immunogen produced by rabbits against the plant glycoprotein HRP is used as a specific neural marker in Drosophila (Paschinger et al., 2009).

As it had been difficult to discriminate between neuronal and non-neuronal cells in the insect nervous system using morphological methods another staining method was developed for studying the postembryonic pattern of neuronal apoptosis. Indeed, the pattern of neuronal apoptosis in the VG of insects during the larval to pupal stages is poorly understood and based on only a few studies on the adult CNS of Drosophila (Truman \& Schwartz, 1984; Truman et al., 1993; Karcavich \& Doe, 2005; Choi et al., 2006; Rezával et al., 2007; Bass et al., 2007; Kato et al., 2009)

Therefore, fluorescent triple staining by TUNEL assay using the antibody anti-horseradish peroxidase (HRP) conjugated with $\mathrm{Cy} 3$, 4',6-diamidino-2-phenylindole (DAPI), and 2'-deoxyuridine 5'-triphosphate (dUTP) conjugated with FITC was used to analyze the pattern of neuronal apoptosis in the twelve VG of the silkworm during the different periods of postembryonic development (Tettamanti \& Malagoli, 2008). The characterization of neuronal apoptosis in the VG was also done by isolation and morphological and biochemical treatments with SOG, TG1 (only numerical changes in apoptotic neurons), TG3, AG4, AG6 and TAG of day-1 or/and day-4 pupae after in vivo injection of $20 \mathrm{E}$ and inhibitors of both protein synthesis and caspase- 8 and -3 into day- 8 th instar larvae or/and day-2 pupae (Ekert et al., 1999).

The results of this study on neuronal apoptosis in silkworm VG were compared with those obtained in a previous study (Kim et al., 2009) using the brains of postembryonic silkworms in order to define the mechanisms of CNS development during the postembryonic life of insects.

It is thought that the fluorescent triple staining method described in this paper, which can be used to identify normal and apoptotic neurons in the silkworm VG, will provide insect neurobiologists with a method of detecting apoptotic neurons in intact neural tissues or in cultures of insect neuronal cells.

\section{MATERIAL AND METHODS}

\section{Experimental animals}

Cold-treated eggs of the silkworm, Bombyx mori, which were kindly provided by the department of agricultural biology at the National Institute of Agricultural Science and Technology (Suwon, Korea), hatched approximately 10 days after incubating them at a temperature of $26.5^{\circ} \mathrm{C}$ and relative humidity of $70 \%$. The 1 st instar larvae were reared on an artificial diet of "Silk-mate" (Nihon Nosan Kogyo, Yokohama, Japan) at $25 \pm$
$1{ }^{\circ} \mathrm{C}$ under a $12 \mathrm{~L}: 12 \mathrm{D}$ cycle and relative humidity of $70 \%$ in a growth chamber (Doori Scientific, Seoul, Korea) (Mizoguchi et al., 2001).

The larvae passed through 5 instars and then metamorphosed into pupae, finally emerging as adults. The first instar larval stage lasted for 4 days, the 2nd 3 days, 3rd 4 days, 4th 4 days, 5th 9 days and pupae 9 days. The majority of 5th instar larvae began to wander on day 5 or 6 and pupated on either day 9 or 10 , depending on the batch. Day-5 and day-9 5th instar larvae were used to monitor the pattern of neuronal apoptosis and for inducing or inhibiting it using $20 \mathrm{E}$ or other chemicals. For monitoring the pattern of neuronal apoptosis or the effects of in vivo injection of $20 \mathrm{E}$ and other chemicals, larvae, pupae and adults $12 \mathrm{~h}$ after hatching or at a particular ecdysis were selected from the rearing stock (Kim et al., 2009).

\section{Fluorescent triple staining of AG7 used to identify non- neuronal cells, normal and apoptotic neurons}

Day-4 pupae collected from the rearing stock were anesthetized by keeping them at $4^{\circ} \mathrm{C}$ for about $15 \mathrm{~min}$ to a half hour, after which they were dissected in a cold room. AG7/8 (TAG) was then isolated under a stereoscope by careful removal of the outermost chitin in $0.1 \mathrm{M}$ phosphate buffer (PB, $\mathrm{pH}$ 7.4), after which AG7 was carefully removed from AG7/8. The isolated AG7 was then fixed in 4\% paraformaldehyde (PFA) in $0.1 \mathrm{M}$ PB for $10 \mathrm{~h}$ at $4^{\circ} \mathrm{C}$ (Nässel, 1996; Kim et al., 2004; Park et al., 2004). The fixed ganglion was washed in $0.1 \mathrm{M} \mathrm{PB}$ for $1 \mathrm{~h}$ and then immersed in $0.01 \mathrm{M}$ phosphate buffer saline with $1 \%$ triton-X (1\% PBST) overnight at $4^{\circ} \mathrm{C}$. After dehydration by passing the sample through an ethanol series of $50 \%$ to $100 \%$, the ganglion was cleared in xylene and embedded in paraffin wax. The ganglion in the paraffin wax block was then sectioned using a microtome (MICRON), after which the $7 \mu \mathrm{m}$ thick sections were mounted on poly-L lysine-coated glass slides and air dried (Leonardi \& Loreto, 2008).

To stain the cytoplasm of the neurons in AG7, the paraffin wax in the ganglion sections was removed using xylene and then the sections were rehydrated using a retrograded ethanol series. The sections were then incubated overnight at room temperature (RT) in blocking solution (5\% normal swine serum in $0.5 \%$ PBST) to reduce non-specific reactions, after which they were immersed in anti-HRP antiserum (Sigma; St. Louis, MO, Product No. P-7899), diluted $1: 10,000$ in blocking solution, for $48 \mathrm{~h}$ at RT. The ganglion sections were washed in $0.1 \%$ PBST and then incubated in goat anti-rabbit conjugated with $\mathrm{Cy} 3$ (Jackson; West Grove, PA, Product No. 111-167-003) in blocking solution in the dark for $48 \mathrm{~h}$ at RT (Loesel et al., 2006).

To label the fragmented DNA in the nuclei of the apoptotic neurons, a TUNEL assay was conducted using a DeadEed ${ }^{\mathrm{TM}}$ Fluorometric TUNEL System Kit (Promega; Product No. G-3250). Briefly, anti-HRP- and Cy3-conjugated secondary antibody-treated ganglion sections were digested with $50 \mu 1$ of proteinase $\mathrm{K}$ solution $(20 \mu \mathrm{g} / \mathrm{ml}$ proteinase $\mathrm{K}$ in EDTA solution buffer) for $10 \mathrm{~min}$ at RT, washed in PBS for $5 \mathrm{~min}$, and then treated in equilibration buffer for 5-10 $\mathrm{min}$ at RT. The ganglion sections were subsequently treated with TdT solution buffer composed of equilibration buffer $(45 \mu \mathrm{l})$ and FITC-conjugated nucleotide mix $(5 \mu \mathrm{l})$ containing dUTP and recombinant terminal deoxynucleotidyl transferase (rTdT; $1 \mu \mathrm{l})$ for $1 \mathrm{~h}$ at $37^{\circ} \mathrm{C}$. The sections were then washed in stop buffer (2XSSC) for 15 min at RT (Tettamanti \& Malagoli, 2008)

To stain the nuclei in the cells of the AG7, ganglion sections were washed three times in PBS for 5 min each, after which they were treated with suitably diluted $(1: 10,000)$ DAPI (Sigma, Product No. P9564; classical nuclear staining dye) for 
12 min and then washed in PBS. One drop of anti-fade reagent (Bio-rad) was then added to the ganglion tissue on glass slides to prevent fluorescent signal fading on exposure to light (Longin et al., 1993), after which the samples were covered with glass coverslips (Bossy-Wetzel \& Green, 2000; Boatright et al., 2003). Sections of AG7 were then examined using a confocal laser scanning microscope (Zeiss LSM 310). The confocal images were used to evaluate the number and magnified features of apoptotic neurons.

\section{Changes in the number of apoptotic neurons in the VG during postembryonic development}

Animals were collected immediately after hatching or at ecdysis throughout postembryonic development (34 days). The collected animals were then dissected and the twelve ganglia in the VNC (SOG, TG1 to TG3, AG1 to AG8) isolated. After isolation the twelve $\mathrm{VG}$ were embedded in wax, sectioned and mounted. The number of sections of the $12 \mathrm{VG}$ was, respectively, 10 to 25 of SOG, 10 to 22 of TG1 to TG3 and 10 to 25 of AG1 to AG8.

After the paraffin wax was removed the sections were rehydrated and treated with HRP antibody conjugated with $\mathrm{Cy} 3$, DAPI and FITC-conjugated nucleotide mix (including dUTP) (See above for details). Magnified images of all the apoptotic neurons in the serial sections of all the ganglia were compared and the number of apoptotic neurons counted. One way ANOVA and chi-square tests $\left(\chi^{2}\right.$-test $)$ were used in the analysis of the changes in the number of apoptotic neurons in the VG.

Injection of 20E, actinomycin $D$ or cycloheximide and inhibitors of caspase-8 and caspase- 3 into 1 day old pupae

The solutions of $0.1,1,5$ or $10 \mu \mathrm{g} / \mathrm{ml}$ of $20 \mathrm{E}$ were prepared from a stock solution, which was made by dissolving $20 \mathrm{E}$ in a very small amount of ethanol and then mixing it with PBS. These solutions were injected through the lateral body wall into the haemolymph of day- 8 th instar larvae and day-2 pupae (with an average body weight of $1 \mathrm{~g}$; physiological level of $20 \mathrm{E}$ is $1 \mu \mathrm{g}$ / body weight $\mathrm{g}$ ) (Sakurai et al., 1998), using a Hamilton syringe (Mitchelle, 1978; Truman, 1993), and the numbers of apoptotic neurons and the cleavage of procaspase- 3 into caspase- 3 in SOG, TG1 (only the number of apoptotic neurons), TG3, AG4, AG6 and TAG of day-1 and day-4 pupae. The same volume $(1 \mu \mathrm{l})$ of each $20 \mathrm{E}$ solution was injected into each insect. The amount of $20 \mathrm{E}$ required to effectively induce neuronal apoptosis in the CNS of silkworm larvae was $10 \mu \mathrm{g} / \mathrm{ml}$. The controls for this experiment were the VG of two different animals injected with the same volumes of either PBS or ethanol (Et-OH).

Day-8 5th instar larvae and day-1 pupae were injected with 1 $\mu 1$ of a solution containing $1 \mu \mathrm{g}$ actinomycin D (ActD, Sigma) or $10 \mu \mathrm{g}$ cycloheximide (CHX, Sigma), which was derived from stock solutions of 0.1 or $10 \mathrm{mg}$ of these chemicals dissolved in $100 \mathrm{mM}$ dimethyl sulfoxide(DMSO). The numbers of apoptotic neurons and cleavage of procaspase- 3 into caspase- 3 in SOG, TG1 (former only), TG3, AG4, AG6 and TAG of day-1 and day-4 pupae were investigated (Hoffman \& Weeks, 2001). The controls in this case were the VG of two different animals injected with the same volumes of either PBS or dimethyl sulfoxide.

The caspase-8 inhibitor (Z-IETD-FMK, Calbiochem) and caspase-3 inhibitor (Ac-DEVD-CMK, Calbiochem) were dissolved in dimethyl sulfoxide at a $100 \mathrm{mM}$ concentration and stored at $-20^{\circ} \mathrm{C}$ until used (Terashima et al., 2000). A volume of $1 \mu \mathrm{l}$ of $50 \mu \mathrm{M}$ of caspase- 8 inhibitor or $25 \mu \mathrm{M}$ of caspase- 3 inhibitor, which were prepared from the stock solution, was injected into the haemolymph of day-8 5th instar larvae and day-1pupae and the number of apoptotic neurons and cleavage of procaspase-3 into caspase-3 in SOG, TG1 (former only), TG3, AG4, AG6 and TAG of day-1 and day-4 pupae were analyzed. In this case the controls were the VG of two different animals injected with the same volumes of either PBS or DMSO.

After injection of $20 \mathrm{E}$, ActD or CHX and inhibitors of caspase- 8 and caspase- 3 into day- 8 th instar larvae and day- 2 pupae, SOG, TG3, AG4 and AG6 to AG8 were carefully isolated from day- 1 and day-4 pupae and then either fluorescent triple stained as described above or subjected to western blotting as described below. The numbers of apoptotic neurons were then recorded.

\section{Western blotting}

The ventral ganglia were also subjected to western blotting to detect various targeted proteins. The VG from day- 8 th instar larvae and day-1 pupae that were only injected with PBS or DMSO were used as controls. Western blotting of all VG, midguts and silk glands from day-4 pupae or specific VG from day-1 and day-4 pupae was done using anti-HRP or anticaspase-3 antibodies. To accomplish this, approximately $60 \mathrm{VG}$, midguts and silk glands were isolated in a cold room, lysed in non-ionic detergent containing RIPA buffer and then sonicated with a sonifier (Bronson). The lysates were centrifuged at $12,000 \mathrm{rpm}$ at a temperature of $4^{\circ} \mathrm{C}$ for $30 \mathrm{~min}$, after which the supernatants were separated by electrophoresis on a $12 \%$ polyacrylamide gel.

The separated proteins were then transferred to nitrocellulose membranes and treated with blocking solution $(5 \%$ skimmed milk in PBST) for $1 \mathrm{~h}$ to reduce non-specific responses. The membranes were then incubated with anti-HRP or antiprocaspase-3 (Cell Signaling; Product No. 9662) and anticleaved caspase-3 (Cell Signaling; Product No. 9661) in blocking solution at $4{ }^{\circ} \mathrm{C}$ and gently shaken for 1 day. The specimens were treated with anti-rabbit swine secondary antibody conjugated with HRP (DAKO; Product No P-0399) for $2 \mathrm{~h}$ at room temperature (Sambrook \& Russell, 2001). The proteins were detected using a Lumminescent Image Analyzer (LAS3000, FUJI film).

\section{RESULTS}

\section{Identification of non-neuronal cells, normal and apoptotic neurons in AG7}

Simple fluorescent triple staining method was used to differentially stain non-neuronal, normal neuronal and apoptotic neuronal cells in twelve ganglia of the VNC at different stages in development from the day-1 1st instar larval stage up to day-1 adults. This method was developed using AG7 and employed anti-HRP conjugated with Cy3 to specifically stain the neuronal cytoplasm, DAPI to stain the nuclei of all the cells and dUTP conjugated with FITC to stain only the fragmented ends of DNA. As shown in Fig. 1, the Cy3 conjugated with anti-HRP antibody stained the cytoplasm of all normal neurons red and DAPI stained their nuclei blue. The nuclei of normal neurons were blue when stained using both TUNEL staining (FITC) and DAPI. Apoptotic neurons had a red cytoplasm and blue nuclei when stained with Cy3 and DAPI, but the color of their nuclei was cyan when stained with both nuclear DAPI and FITC. This change from blue to cyan was the most important criterion for determining whether the neurons were apoptotic or not. This change is evident in the magnified pictures of two normal neurons 

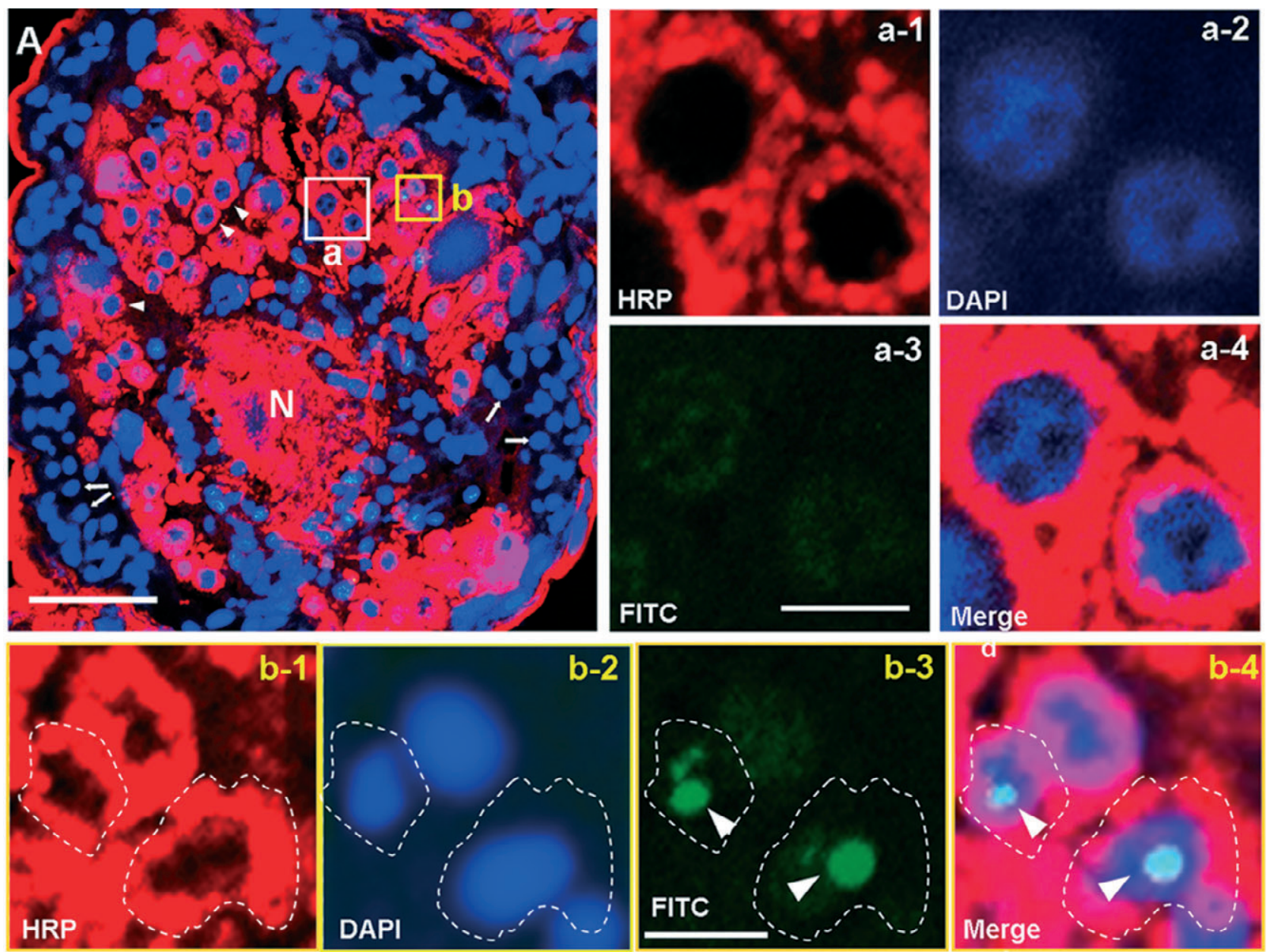

B
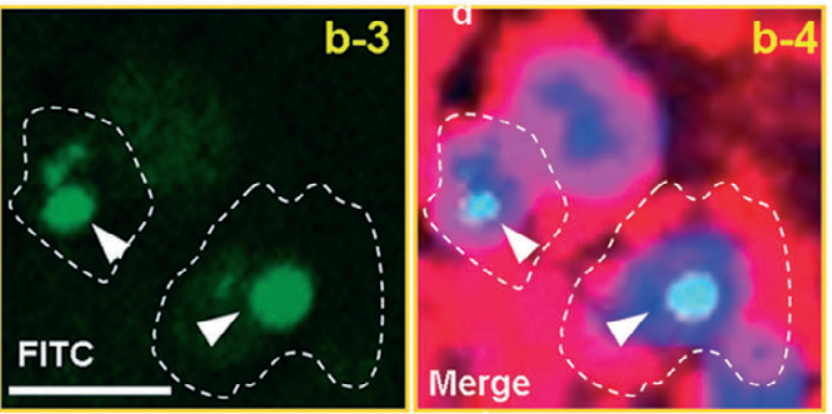

VG midgut silk gland

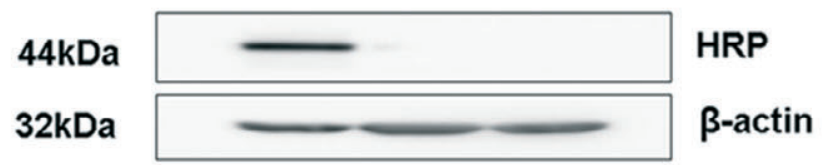

Fig. 1. Fluorescent triple staining of non-neuronal cells, normal and apoptotic neurons in AG7 of silkworm. The cytoplasm of all neurons stained red, the nuclei of all normal cells stained blue, and the fragmented DNA within the nuclei of apoptotic neurons stained in cyan. (A) General features of approximately 65 normal neurons (some indicated by small white arrowheads and cell bodies of two representative normal neurons boxed in "a") and two apoptotic neuronal cells (boxed in "b") in one of 25 sections of AG7 from a B. mori day-4 pupa. All glial cells had nuclei (some indicated by thin white arrows) the edges of which mainly stained blue and unstained cytoplasm. $\mathrm{N}$ - neuropil. Scale bar $=60 \mu \mathrm{m}$. (a-1, 2, 3, 4) Magnification of two representative normal neuronal cell bodies boxed in "a" of Fig. 1A. (a-1) Two normal neuronal cell bodies with red cytoplasm stained with anti-HRP conjugated with Cy3. (a-2) Nuclei of two normal neuronal cell bodies stained blue by DAPI. (a-3) Nuclei of two normal neuronal cell bodies stained green by FITC. Scale bar $=20 \mu \mathrm{m}$ and indicates the magnification of a-1, a-2 and a-4. (a-4) Two normal neuronal cell bodies with their cytoplasm stained red and nuclei blue due to combined staining by HRP, DAPI and FITC. (b-1, 2, 3, 4) Magnified image of two apoptotic neuronal cell bodies (outlined by the dotted line) boxed in "b" of Fig. 1A. Nuclear condensation and fragmentation in apoptotic neuronal cell bodies appeared green due to staining by FITC, but in cyan when stained with DAPI and FITC. The scale bar in $b-3=20 \mu \mathrm{m}$ and the magnification of b-1, b-2 and b-4. (b-1) Two apoptotic (outlined) and one normal neuronal cell body with red cytoplasm due to staining with anti-HRP and Cy3. (b-2) The nuclei of two apoptotic neuronal cell bodies (outlined) and one normal neuronal cell body stained blue with DAPI. Nuclear condensation and fragmentation (white arrowheads) in two apoptotic neuronal cell bodies (outlined) stained green by FITC (b-3) or in cyan when stained with DAPI and FITC (b-4). (B) Western blotting of the VG, midguts and silk glands of $B$. mori day-4 pupae with anti-HRP antibody revealed the production of HRP by the VG but not by the midguts or silk glands.

(Figs 1a-1, 1a-2, 1a-3, 1a-4) and two apoptotic neurons (Figs 1b-1, 1b-2, 1b-3, 1b-4), and indicates that cyan staining of the nuclei of apoptotic neurons results from the merging of the nuclear DAPI with FITC during nuclear condensation and fragmentation.

Because all glial cells have blue nuclei and unstained cytoplasm (small white arrows in Fig. 1A) they are 

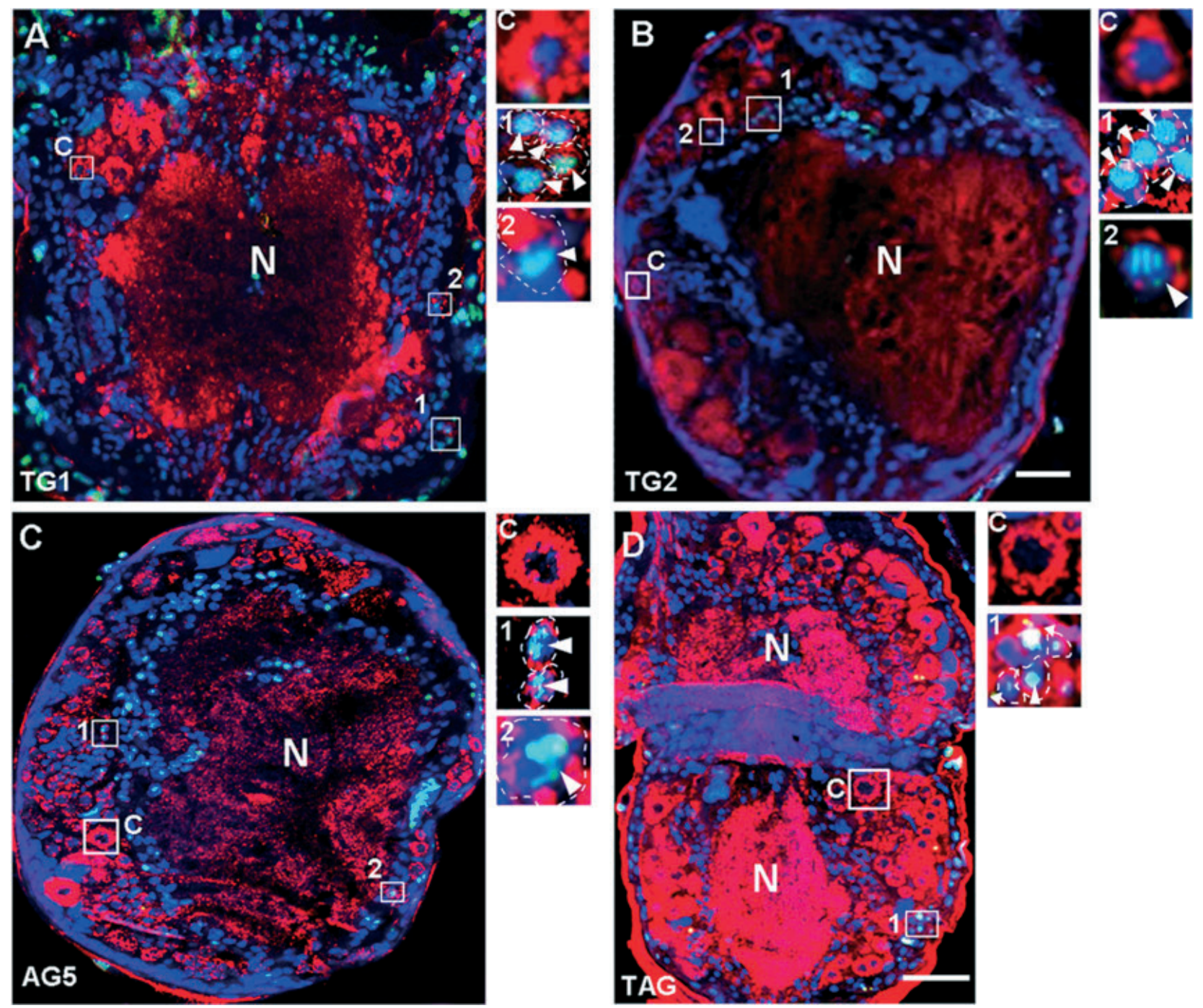

Fig. 2. Apoptotic neurons in four representative sections of the SOG of a day-5 pupa, TG2 of day-1 pupa, AG5 of day-4 pupa and TAG of day-4 pupa following fluorescent triple staining. (A) Four apoptotic neurons indicated by box 1 on one of the 17 sections of TG1 of a day-1 pupa. (B) One of the 15 sections of TG2 of a day-1 pupa showing five apoptotic neurons (outlined or not) in box 1 and 2. (C) Three apoptotic neurons in box 1 and 2, in one of 15 sections of AG5 of a day-4 pupa. (D) One of 21 sections of TAG of a day-4 pupa with three apoptotic neurons in box 1 and 2. To the right of the four representative ganglia, the apoptotic neurons show nuclear condensation and fragmentation (arrowheads) when the features of the individual neurons (1, 2 or 3), were compared with those of normal neurons (C). Approximately 43 apoptotic neurons were found in the 17 sections of TG1 of day-1 pupae, approximately 56 in TG2, approximately 79 in AG5 of day-4 pupae and approximately 225 in TAG of day-4 pupae. All scale bars $=30 \mu \mathrm{m}$.

clearly different from normal or apoptotic neurons. In addition, approximately 65 normal neurons with red cytoplasm and blue nuclei (two normal neurons are marked by small white arrowheads in box "a" of Fig. 1A) were observed in one of the 25 sections of AG7 (Fig. 1A). There are approximately 2 apoptotic neurons with red cytoplasm and cyan nuclei in the same section (box " $b$ " of Fig. 1A). These normal and apoptotic neurons were located in the immediate surroundings (cortex) of neurophils at the centers of the ganglia (Figs 1A and 2).

The western blotting of all VG of day-4 pupae, using the anti-HRP antibody, gave positive results, but not the western blotting of the midgut and silk gland (Fig. 1B). This indicates that only neurons in the VG produce the insect neuronal marker, HRP $(44 \mathrm{kDa})$.

\section{Changes in the numbers of apoptotic neurons in the ventral ganglia during postembryonic development}

That the nuclei of some VG neurons were a cyan color (white arrowheads in boxes 1 and 2 in Figs $2 \mathrm{~A}, \mathrm{~B}, \mathrm{C}$ ), due to the merging of the effects of staining with nuclear DAPI and FITC, indicates that some of them were in the process of apoptosis and were undergoing nuclear condensation and/or fragmentation. This enabled the number of apoptotic neurons in the twelve VG to be determined at different stages during postembryonic development (Figs 3A, B).

During postembryonic development there were three patterns in the changes in the numbers of apoptotic neurons observed in the twelve ganglia of the VNC: one for SOG, one for TG1 to TG3 and AG1 to AG4, and one for AG5 to AG8. As shown in Fig. 3A, the number of apop- 

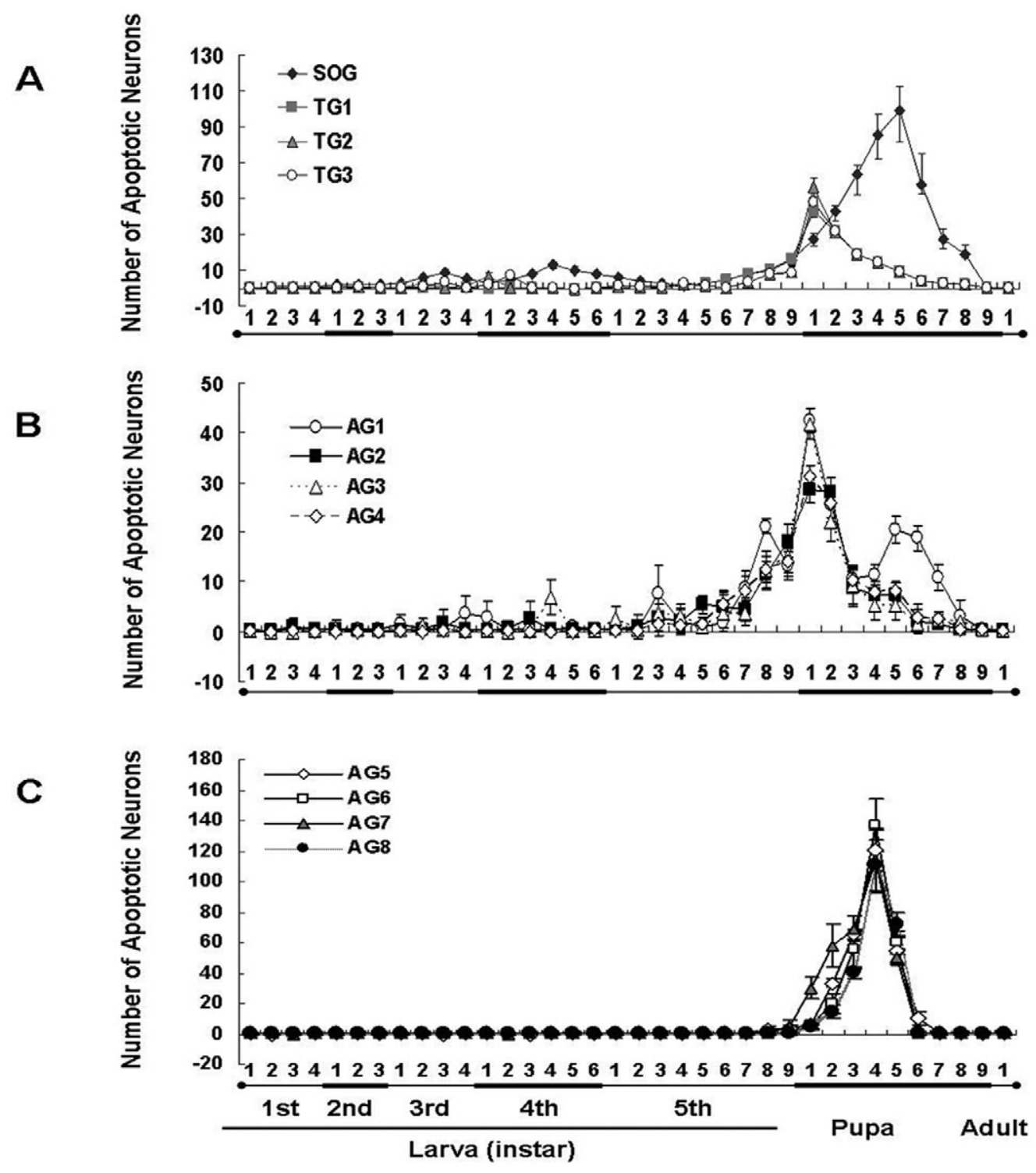

Postembryonic Stages

Fig. 3. The patterns of neuronal apoptosis in the SOG, three TG and eight AG at the larval, pupal and adult stages. (A) Patterns of neuronal apoptosis in SOG, TG1, TG2 and TG3. The greatest numbers of apoptotic neurons were recorded in SOG in a day-4 pupa and in TG1 to TG3 immediately after pupation. During the larval stages, a small number of apoptotic neurons were found in SOG in the middle of the 4th instar and at the end of 5th instar, and the number of these neurons was greater in TG1 to TG3 at the end of the 5th instar. (B) Patterns of neuronal apoptosis in AG1 to AG8. The greatest number of apoptotic neurons was recorded in AG1 to AG4 immediately after pupation and in AG5 to AG8 in a day-4 pupa. An increased number of apoptotic neurons were recorded in AG1 to AG4 near or at the end of the 5th instar. (C) Patterns of neuronal apoptosis in AG5 to AG8. The greatest numbers of apoptotic neurons were recorded in AG5 to AG8 of a day-4 pupa. There were few apoptotic neurons recorded in AG5 to AG8 during the larval stages. Results for the different groups within each stage were compared using a one way ANOVA $(P<0.05)$.

totic neurons in SOG increased slightly around day-4 in the 4th instar larva and then decreased. However, there was a significant increase in the number of apoptotic neurons near the end of larval life or during the wandering stages and after pupation, with the peak occurring in day-5 pupae. In TG1 to TG3 and AG1 to AG4, fewer neurons underwent apoptosis during most larval stages, but apoptotic neurons began to increase near the end of the larval period.

This increase was followed by a maximum in day-1 pupae and then a rapid decrease. However, the decrease in the number of apoptotic neurons in AG1 continued until day-9 (Fig. 3B). In AG5 to AG8, neuronal apoptosis began at the end of larval life or immediately after pupation and then gradually increased to a maximum on day-4. Large apoptotic neurons, with diameters of more than 20 $\mu \mathrm{m}$, were present in the cortex of TG1, TG2 and AG5 (see magnified neurons in Figs 2A-2, 2B-2, 2C-2). Apoptotic neurons were not observed in the $\mathrm{VG}$ on day-1 of the 1st larval stage, day- 9 of the pupal stage or day-1 of the adult stage. 
Neuronal apoptosis in SOG, TG1, TG3, AG4, and AG6 to AG8 of day-1 pupae injected with $20 \mathrm{E}$ and other chemicals on day- 8 of the 5 th larval instar

The changes in the numbers of apoptotic neurons and the cleavage of procaspase- 3 into active caspase- 3 after injection of $20 \mathrm{E}$ and other chemicals were evaluated in SOG, TG1, TG3, AG4 and AG6 to AG8. As indicate above, SOG showed one pattern of neuronal apoptosis, TG1, TG3 and AG4 a second and AG6 to AG8 a third.

Two days after in vivo injection of day- 8 th instar larvae with $20 \mathrm{E}$, changes in the numbers of apoptotic neurons and the cleavage of procaspase-3 $(35 \mathrm{kDa})$ into active caspase-3 $(17 \mathrm{kDa})$ were investigated in SOG, TG1, TG3, AG4 and AG6 to AG8. In response to the $20 \mathrm{E}$ injection the number of apoptotic neurons increased in SOG and AG6 to AG8, but only slightly in TG1. In contrast the number of apoptotic neurons decreased in TG3 and AG4 (Fig. 4A). Furthermore, western blot analysis of SOG, TG3, AG4, AG6 and TAG for procaspase-3 cleavage following the injection of $20 \mathrm{E}$ revealed that the cleavage increased in SOG, AG6 and TAG (Fig. 4B). However, increasing the titre of $20 \mathrm{E}$ injected did not increase the cleavage of procaspase- 3 . These findings suggest that increasing the titre of $20 \mathrm{E}$ had a different effect on neuronal apoptosis in SOG, TG1, and AG6 to AG8 from on TG3 and AG4.

Changes in the numbers of apoptotic neurons and the cleavage of procaspase- 3 into active caspase- $348 \mathrm{~h}$ after in vivo injection of ActD or CHX into day-8 5th instar larvae and day-2 pupae were monitored in SOG, TG1 (only the former), TG3, AG4, AG6 and TAG. The great reduction in the number of apoptotic neurons recorded indicates that neuronal apoptosis was significantly inhibited in these ganglia (Figs 5A-1, -2). Furthermore, the western blot analysis of the cleavage of procaspase- 3 into caspase-3 in SOG, TG3, AG4, AG6 and TAG after injection of ActD or $\mathrm{CHX}$ revealed that procaspase- 3 cleavage was inhibited in SOG, AG6 and TAG but the injection of $20 \mathrm{E}$ had little effect on cleavage of procaspase-3 in TG3 and AG4 (Figs 5B-1, -2). These findings suggest that neuronal apoptosis in SOG, AG6 and TAG is associated with the synthesis of a new protein, but might not be triggered in TG3 and AG4 by the synthesis of procaspase-3.

Changes in both the number of apoptotic neurons and the cleavage of procaspase- 3 into caspase- $348 \mathrm{~h}$ after in vivo injection of inhibitors of caspase- 8 , and -3 into day- 8 5 th instar larvae and day-2 pupae were traced in SOG, TG1 (only the former), TG3, AG4, and AG6 to AG8. Neuronal apoptosis was clearly inhibited in SOG and AG6 to AG8 by injection of caspase- 8 and -3 , but although slightly reduced, there was not a significant reduction in the number of apoptotic neurons in TG1, TG3 and AG4, especially in day-1 pupae (Fig. 6A).

Western blot analysis conducted to evaluate the role of caspase-8, -3 and -9 in neuronal apoptosis in SOG, TG3, AG4, AG6 and TAG revealed that procaspase-3 cleavage was inhibited in SOG, AG6 and TAG by the inhibitors caspase- 8 and caspase- 3 but not caspase-9, which is

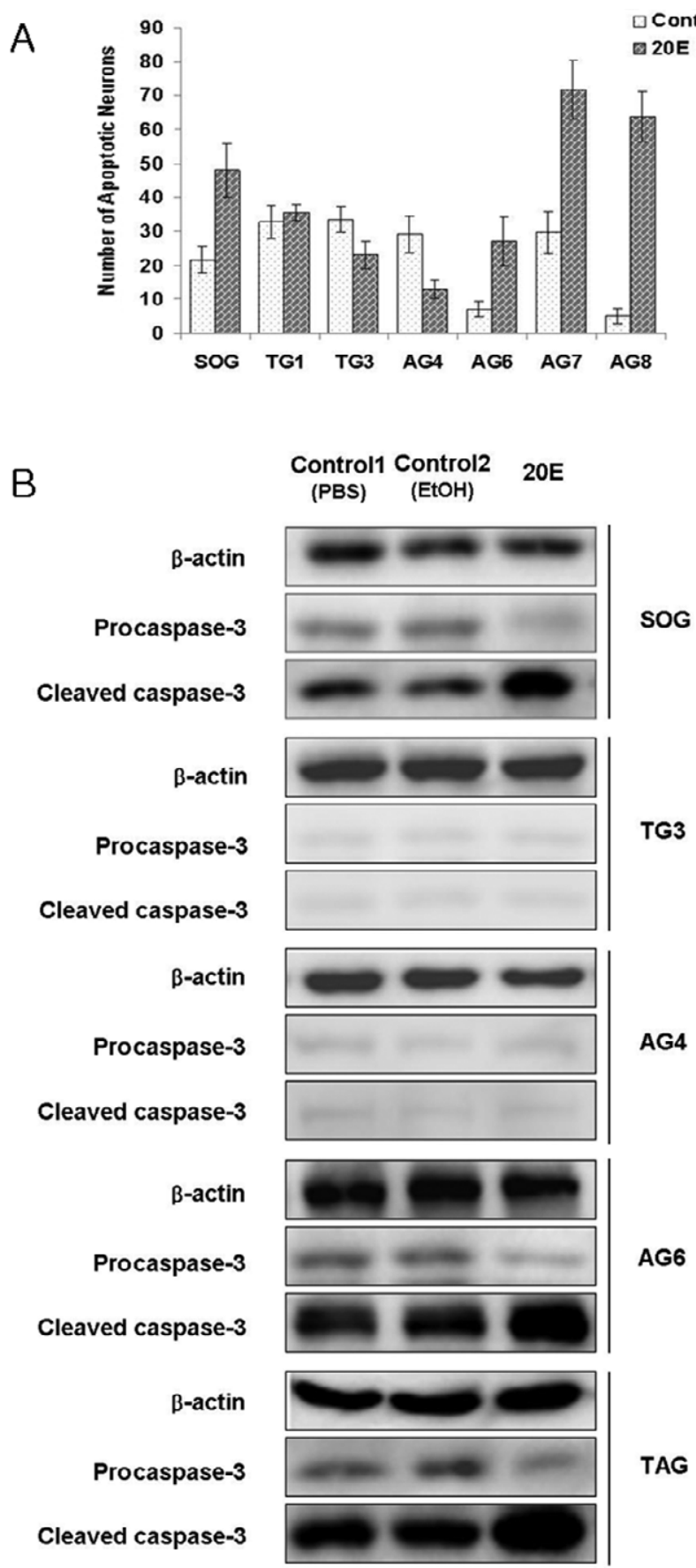

Fig. 4. The number of apoptotic neurons and cleavage of inactive procaspase- 3 into active caspase- 3 in SOG, TG1 (only the former), TG3, AG4, and AG6 to AG8 (or TAG) of day-1 pupae after in vivo injection of $20 \mathrm{E}$ into day-8 5 th instar larvae. (A) The number of apoptotic neurons in SOG, TG1, TG3, AG4, and AG6 to AG8 after the injection of 20E. In SOG and AG6 to AG8, the number increased significantly following the injection, whereas there was only a slight increase in TG1. Conversely, there was a decrease in TG3 and AG4. Differences among groups were determined using $\chi^{2}$-tests $(P<0.05)$. (B) Western blotting to determine if the injection of $20 \mathrm{E}$ into day- 8 5 th instar larvae caused procaspase- 3 to be cleaved into caspase-3 in SOG, TG3, AG4, AG6 and TAG of day-1 pupae. A substantial increase in cleavage of procaspase- 3 into caspase- 3 was recorded in SOG, AG6 and TAG, while the injection had little effect on the synthesis of procaspase- 3 and cleavage of procaspase- 3 in TG3 and AG4. 

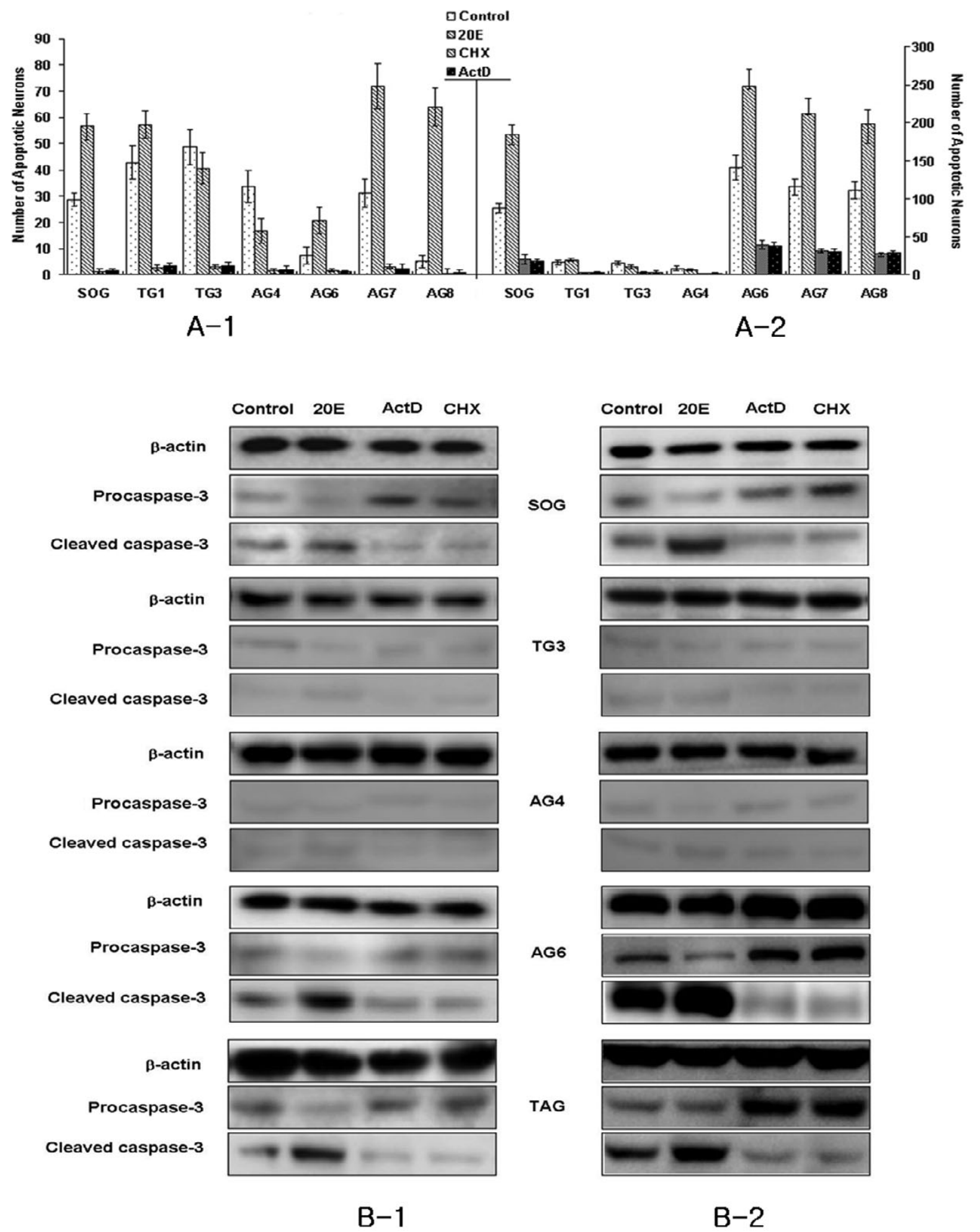

Fig. 5. The number of apoptotic neurons and cleavage of procaspase- 3 into caspase- 3 in SOG, TG1 (only the former), TG3, AG4, AG6 and TAG of day-1 and day-4 pupae after in vivo injection of ActD or CHX into day-8 5th instar larvae and day-2 pupae. Changes in the number of apoptotic neurons in SOG, TG1, TG3, AG4 and AG6 to AG8 in day-1 pupae (A-1) and day-4 pupae (A-2) recorded after the injection of ActD or CHX. There was a significant reduction in the number of apoptotic neurons in all the VG tested. Differences among groups were determined by $\chi^{2}$-tests $(p<0.05)$. Western blotting determined if the cleavage of procaspase-3 into caspase-3 is inhibited in SOG, TG3, AG4, AG6 and TAG of day-1 pupae (B-1) and day-4 pupae (B-2) by injection of either ActD or CHX. These injections into both day-1 and day-4 pupae resulted in the inhibition of procaspase- 3 cleavage in SOG, AG6 and TAG, but in TG3 and AG4 there was little change in the level of procaspase-3 and no cleavage of procaspase-3 into caspase-3. 


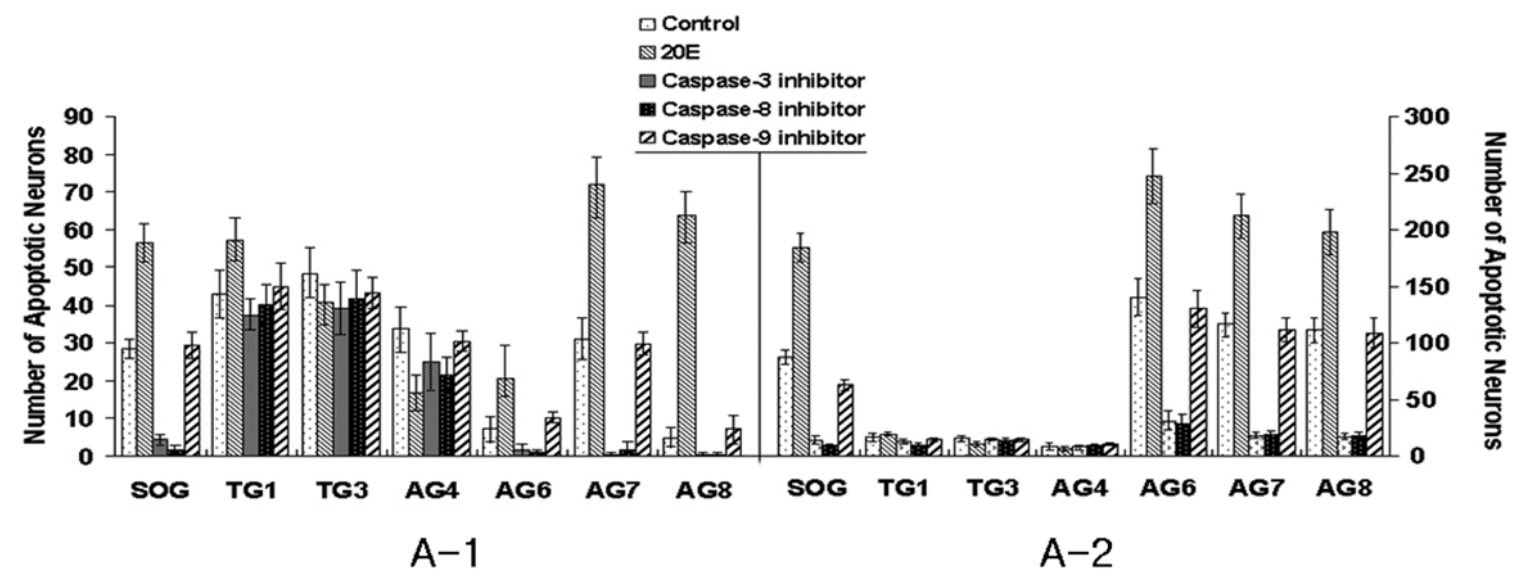

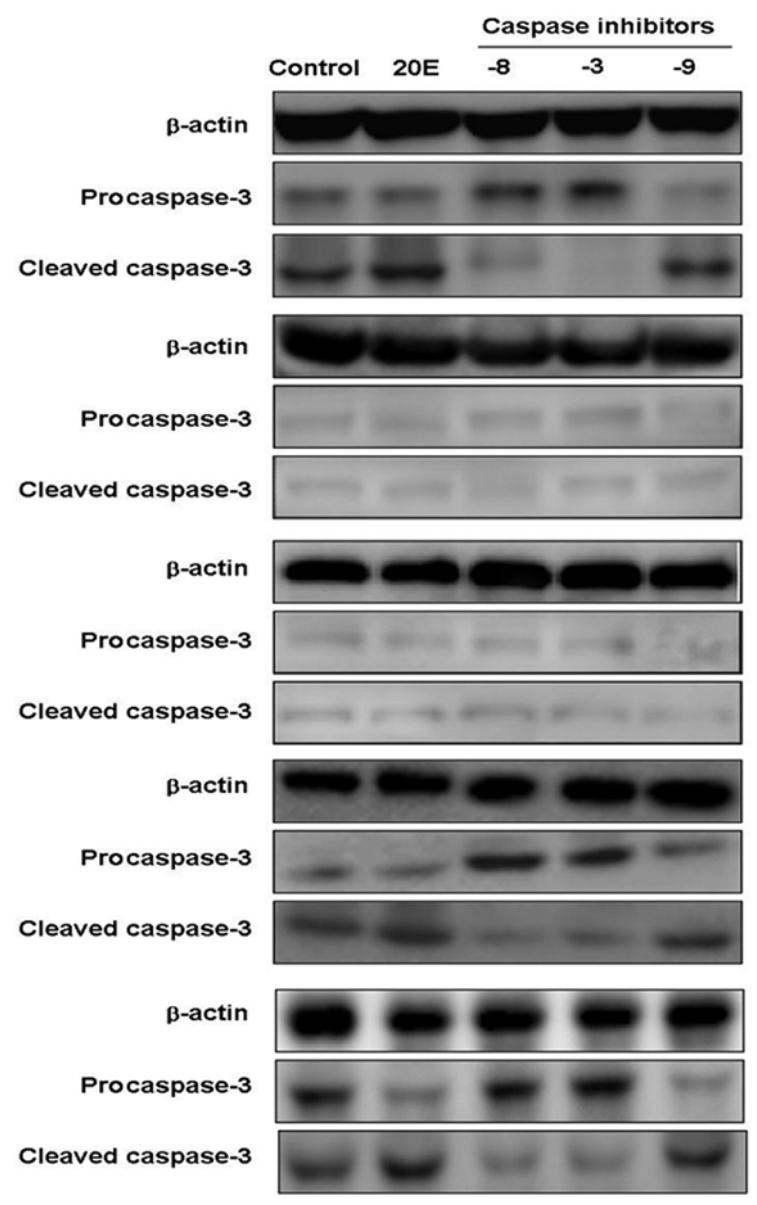

B-1
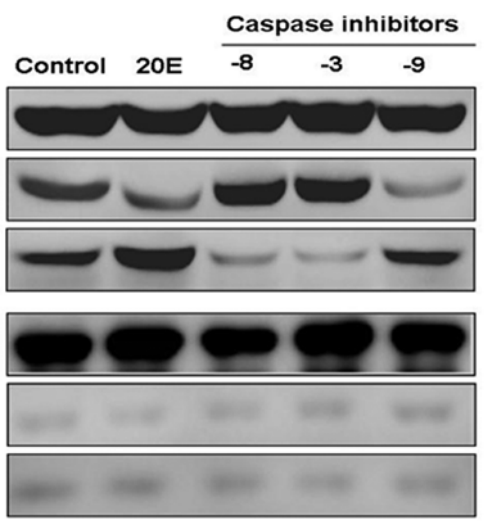

AG4
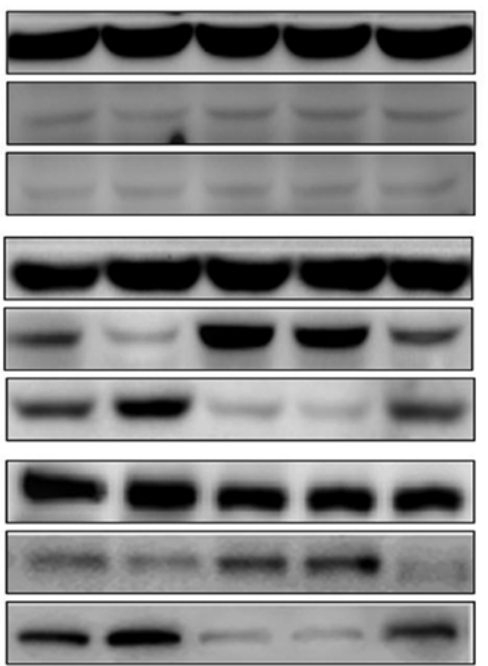

B-2

Fig. 6. Number of apoptotic neurons and cleavage of procaspase-3 into caspase-3 in SOG, TG1 (only the former), TG3, AG4, AG6 and TAG of day-1 and day-4 pupae after in vivo injection of three kinds of caspase (caspase- $8,-3$ and -9 ) inhibitors into day-8 5th instar larvae and day-2 pupae. The number of apoptotic neurons was substantially reduced in SOG and AG6 to AG8 in both day-1 pupae (A-1) and day-4 pupae (A-2) after the injection, whereas in TG1, TG3 and AG4 it resulted in an insignificant decrease. Results were compared using $\chi^{2}$-tests $(p<0.05)$. Western blotting indicated that the cleavage of procaspase-3 in SOG, TG3, AG4, AG6 and TAG of day-1pupae (B-1) and day-4 pupae (B-2) was only significantly inhibited by injection of caspase-8 and -3 inhibitors in SOG, AG6 and TAG,. The injection of the same inhibitors, however, resulted in little quantitative change in procaspase- 3 and no cleavage of procaspase-3 into caspase-3 in TG3 and AG4.

known to participate in the mitochondria-dependentsignal transduction pathway (Fig. 6B).

In TG3 and AG4, however, there were little quantitative change in procaspase- 3 and no cleavage of procaspase- 3 into caspase- 3 after injection of caspase- 8 and -3 , as in the two controls.

These results suggest that, during some of the postembryonic stages, most of neuronal apoptosis in SOG, AG6 
and TAG is induced by a signal transduction pathway in which both caspase- 8 and -3 are involved, whereas for most of neurons that undergo apoptosis in TG3 and AG4 these caspases are not involved. Therefore, the mechanism(s) of neuronal apoptosis in TG3 and AG4 of insects needs further study.

\section{DISCUSSION}

\section{Discrimination of non-neuronal cells, normal and apoptotic neurons by fluorescent triple staining and changes in the numbers of apoptotic neurons in VG during postembryonic development}

A simple fluorescent double staining method has been used to distinguish neuronal cells from non-neuronal cells in the brain of the cockroach (Leucophaea maderae) and the TG of the locust (Locusta migratoria) (Loesel et al., 2006). The fluorescent triple staining used here detected apoptotic neurons in the VG of silkworm. This fluorescent triple staining with TUNEL assay, using antihorseradish peroxidase conjugated with $\mathrm{Cy} 3$, 4',6-diamidino-2-phenylindole and dUTP combined with FITC, successfully discriminated between non-neuronal cells, normal and apoptotic neurons in the VG of silkworm.

The cytoplasm of normal and apoptotic cells stained red and the nuclei of normal neuronal and glial cells blue. The nuclei of apoptotic neurons stained green. When nuclear DAPI was used in combination with FITC, the nuclei of normal neurons stained blue, but those of apoptotic neurons stained in cyan. As shown in Figs 1 and 2, double stained normal neurons had red cytoplasm and blue nuclei; whereas the triple stained apoptotic neurons had red cytoplasm and cyan nuclei.

The fluorescent triple staining made it possible to analyze changes in the numbers of apoptotic neurons in SOG, TG1 to TG3, AG1 and AG8 during postembryonic development from day-1 of the 1 st instar to day-1 of the adult phase in silkworm. This revealed that neuronal apoptosis occurred in a specific pattern in time in each ganglion. Specifically, peaks in the number of apoptotic neurons were observed on day-5 in SOG, day-1 in TG1 to TG3 and AG1 to AG4, and day-3 in AG5 to AG8 (Fig. $3)$. The incidence of neuronal apoptosis in TG1 to TG3 and AG1 to AG4 was lower than in SOG and AG5 to AG8.

This pattern of neuronal apoptosis in TG1 to TG3 and AG1 and AG4 in the silkworm is similar to that in the $\mathrm{VG}$, of $D$. melanogaster in which dying neurons peaked approximately $6 \mathrm{~h}$ and persisted for $24 \mathrm{~h}$ after pupation (Truman et al., 1993). Some of the large neurons are presumably larval neuroblasts that are dying (Fig. 2) as previously suggested for $D$. melanogaster.

In our previous study of neuronal apoptosis in the brains of postembryonic stages of silkworm (Kim et al., 2009), peak numbers of apoptotic neurons were detected on day- 4 in 4 th instar larvae and day-9 in 5th instar larvae and day-4 in pupae. No apoptotic neurons were detected on day-1 in 1st instar larvae, on day-7 in pupae or on day- 1 in adults. These results indicate that different types of brain neurons show three peaks in neuronal apoptosis in response to the three different titres of $20 \mathrm{E}$ injected into day-2.5 4th instar larvae, day-7.8 5th instar larvae and day-2.5 pupae (Mizoguchi et al., 2001). However, there was only one peak in apoptosis of the neurons in each of the VG of postembryonic silkworms. Therefore, the pattern of neuronal apoptosis in the VG is completely different from that in the brain.

\section{Neuronal apoptosis recorded in SOG, TG1, TG3, AG4, and AG6 to AG8 after injection of $20 \mathrm{E}$ or/and other chemicals into day-8 5 th instar larvae or/and day-2 pupae}

During metamorphic reorganization of insect CNS, fluctuation in the $20 \mathrm{E}$ titre in haemolymph evokes neuronal apoptosis, cell proliferation and maturation, and remodelling of larval neurons into adult forms (Schubiger et al., 1998). In the postembryonic brains of silkworms increasing the titre of $20 \mathrm{E}$ in the haemolymph by injection triggered a significant increase in neuronal apoptosis.

Injection of $20 \mathrm{E}$ into the pupae of M. sexta (Truman \& Schwartz, 1984) and larvae of D. melanogaster (Draizen et al., 1999) induced apoptosis of abdominal motoneurons and interneurons or specific neurons in the VG. In $M$. sexta, increasing the concentration of $20 \mathrm{E}$ induces in primary culture the apoptosis of the accessory planta retractor (APR) motoneurons (APR5 and APR6) isolated from AG5 and AG6 of prepupae, while lowering the concentration induces apoptosis of APR motoneurons isolated from AG3 and AG4 of the pupae (Streichert et al., 1997; Hoffman \& Weeks, 1998, 2001; Zee \& Weeks, 2001).

In day-1 pupae of the silkworm, which was preceded by an intermediate peak of $20 \mathrm{E}$ between day- 7 and day- 8 of the 5th instar (Mizoguchi et al., 2001), all VG tested showed three levels of neuronal apoptosis in response to injection of $20 \mathrm{E}$ into day- 8 th instar larvae: a significant increase in the number of apoptotic neurons in SOG and AG6 to AG8, slight increase in TG1 and decrease in TG3 and AG4 (Fig. 4A). As shown in Fig. 4B, western blotting revealed a remarkable decrease in the quantity of procaspase- 3 and cleavage of procaspase-3 into caspase-3. The same results were recorded in the two controls.

Taken together, these findings suggest that most of the neurons that died in the ventral ganglia tested underwent apoptosis in response to an increase in the titre of $20 \mathrm{E}$ in the haemolymph, as reported here for silkworm SOG and AG6 to TAG, silkworm brain (Kim et al., 2009) and $M$. sexta APR(5) and APR(6) (Zee \& Weeks, 2001), but some neurons, such as those in TG3 and AG4, do not.

In earlier studies (Hoffman \& Weeks, 2001; Zee \& Weeks, 2001) it was demonstrated that M. sexta VGs respond differently to the same hormonal conditions. The APR (4) shows a remarkable apoptotic response to an increase in 20E, whereas it induces a growth of neurites in APR (6). These phenomena suggest that same physiological cues can induce different responses in different VG. 
As shown in Fig. 5, most of the neurons that normally showed apoptosis in SOG, TG1, TG3, and AG4 to AG8 of day-1 and day-4 pupae exhibited no apoptosis after ActD or CHX was injected into day-8 5th instar larvae and day-2 pupae. This suggests that the neuronal apoptosis triggered in these ganglia by the increase in $20 \mathrm{E}$ may be induced by the synthesis of a new protein initiated by $20 \mathrm{E}$, as in the brain of silkworm (Kim et al., 2009).

Expression of procaspase- 3 and its subsequent cleavage into caspase-3, which is involved in one of the final steps in the apoptotic process (Mehmet, 2000), is initiated by distinct signals in animal species, including an increase in the titre of $20 \mathrm{E}$ in the brain of silkworms (Kim et al., 2009), status epilepticus in rats (Narkilahti et al., 2003) and alcohol in mice (Young et al., 2003). In the present study, cleavage of inactive procaspases- 8 and -3 into active caspases- 8 and -3 in SOG, TG3, AG4, AG6 and TAG of day- 1 and day- 4 pupae was initiated by injecting $20 \mathrm{E}$ into the haemolymph. The results recorded here for silkworm VG are very similar to those obtained for silkworm brains, but there was no cleavage of the procaspases in TG3 and AG4 induced by the increase in the titre of $20 \mathrm{E}$ in the haemolymph (Fig. 6).

Therefore, it should be noted that neurons in TG3 and AG4 of day-1 and day-4 pupae might need to synthesize proteins in order to undergo apoptosis, and apoptosis of neurons in these two VG might occur in response to signals other than an increase in the titre of $20 \mathrm{E}$ in the haemolymph. The biochemical characteristics of neuronal apoptosis in silkworm TG3 and AG4 need further study.

In conclusion, different patterns of neuronal apoptosis in the VG of postembryonic silkworms were revealed by fluorescent triple staining that involved a TUNEL assay using anti-HRP conjugated with Cy3, DAPI and dUTP conjugated with FITC. Both the pattern of neuronal apoptosis in the VG during metamorphosis and the method of fluorescent triple staining used will provide insect neurobiologists with important knowledge and a means of studying apoptotic neurons in intact neural tissues or cultures of insect neuronal cells. It is possible to discriminate between apoptotic and normal neurons in intact insect neural tissues or in culture by this method. Data on the pattern and biochemical characteristics of neuronal apoptosis in the postembryonic stages will be valuable in defining the development of the CNS of insects during metamorphosis.

ACKNOWLEDGEMENT. This study was funded by a grant to Bong Hee Lee in 2008 from the Korea University.

\section{REFERENCES}

Bass B.P., Cullen K. \& McCall K. 2007: The axon guidance gene lola is required for programmed cell death in the Drosophila ovary. Dev. Biol. 304: 771-785.

Bate M. \& Martinez-Arias A. (eds) 1993: The Development of Drosophila melanogaster. Vol. 2. CSHL Press, Cold Spring Harbor, NY

Boatright K.M., Renatus M., Scott F.L., Sperandio S., Shin H., Pedersem I.M., Ricci J.E., Edris W.A., Sutherlin D.P., Green D.R. \& Salvesen G.S. 2003: A unified model for apical caspase activation. Mol. Cell 11: 529-541.
Bossy-Wetzel E. \& Green D.R. 2000: Detection of apoptosis: annexin V laveling. Meth. Enzymol. 332: 15-18.

Bullock T.H. \& Horridge G.A. 1965: Structure and Function in the Nervous Systems of Invertebrates. Vol. 1. Freeman, London.

Choi Y.J., Lee G. \& Park J.H. 2006: Programmed cell death mechanisms of identifiable peptidergic neurons in Drosophila melanogaster. Development 133: 2223-2232.

Draizen T.A., Ewer J. \& Robinow S. 1999: Genetic and hormonal regulation of the death of peptidergic neurons in the Drosophila central nervous system. J. Neurobiol. 38: 455-465.

Ekert P.G., Silke J. \& Vaux D.L. 1999: Caspase inhibitors. Cell Death Differ. 6: 1081-1086.

Hirashima A., Yamaj H., Yoshizawa T., Kuwano E. \& Eto M. 2007: Effect of tyramine and stress on sex-pheromone production in the pre- and post-mating silkworm moth, Bombyx mori. J. Insect Physiol. 53: 1242-1249.

Hoffman K.L. \& Weeks J.C. 1998: Programmed cell death of an identified motoneuron in vitro: Temporal requirements for steroid exposure and protein synthesis. J. Neurobiol. 35: 300-322.

HoffmAn K.L. \& WeEks J.C. 2001: Role of caspases and mitochondria in the steroid-induced programmed cell death of a motoneuron during metamorphosis. Dev. Biol. 229: 517-536.

Karcavich R. \& Doe C. 2005: Drosophila neuroblast 7-3 cell lineage: A model system for studying programmed cell death, Notch/Numb signaling, and sequential specification of ganglion mother cell identity. J. Comp. Neurol. 481: 240-251.

Kato K., Awasaki T. \& Ito K. 2009: Neuronal programmed cell death induces glial cell division in the adult Drosophila brain. Development 136: 51-59.

Kim J.H., PARK C.W., Song H.Y., Kim M.Y., Chol C.O. \& LeE B.H. 2004: Effects of brain-derived neurotrophic factor on neurite growth of deutocerebral neurons in brain of the silk moth Bombyx mori. Entomol. Res. 34: 113-122.

Kim M.Y., Кim K.M., Choi C.O., Song H.W., Lee C.S., Kim B.W., Kang P.D., Jeon S.H., Cho B.P., Bae Y.J., Ko Y.G. \& LeE B.H. 2009: Apoptosis of neuronal cells in the brains of postembryonic silkworms Bombyx mori (Lepidoptera: Bombycidae). Eur. J. Entomol. 106: 335-345.

LEONARDI R. \& LORETO C. 2008: Immunohistochemical location of tissue inhibitor of matrix metalloprotease-1 (TIMP-1) in human carious dentine. Acta. Histochem. doi:10.1016/j.acthis. 2008.09.005

LeVINE R.B. \& TRuman J.W. 1985: Dendritic reorganization of abdominal motoneurons during metamorphosis of the moth, Manduca sexta. J. Neurosci. 5: 2424-2431.

Loesel R., Weigel S. \& Braunig P. 2006: A simple fluorescent double staining method for distinguishing neuronal from nonneuronal cells in the insect central nervous system. J. Neurosci. Meth. 155: 202-206.

Longin A., Souchier C., Frrench M. \& Bryon P.A. 1993: Comparison of anti-fading agents used in fluorescence microscopy: image analysis and laser confocal microscopy study. J. Histochem. Cytochem. 41: 1833-1840.

Menmet H. 2000: Caspases find a new place to hide. Nature 403: $20-30$.

Mitchell H.K. 1978: An apparatus for microinjection of Drosophila. In Ashburner M. \& Wright T.R.F. (eds): The Genetics and Biology of Drosophila, 2a. Academic Press, New York, pp. 150-155.

Mitsumasu K., Ohta H., Tsuchihara K., Asaoka K., Ozoe Y., Nimi T., Yamashita O. \& Yaginuma T. 2008: Molecular cloning and characterization of cDNAs encoding dopamine 
receptor-1 and -2 from brain-suboesophageal ganglion of the silkworm, Bombyx mori. Insect Mol. Biol. 17: 185-195.

Mizoguchi A., Ohashi Y., Hosoda K., Ishibashi J. \& Kataoka H. 2001: Developmental profile of the changes in the prothoracicotropic hormone titer in hemolymph of the silkworm Bombyx mori: correlation with ecdysteroid secretion. Insect Biochem. Mol. Biol. 31: 349-358.

Narkilahti S., Pirttilä T.J., Lukasiuk K., Tuunanen J. \& PitKÄNEN A. 2003: Expression and activation of caspase 3 following status epilepticus in the rat. Eur. J. Neurosci. 18: $1486-1496$.

NÄSSEL D.R. 1996: Advances in the immunocytochemical localization of neuroactive substances in the insect nervous system. J. Neurosci. Meth. 69: 3-23.

Niven J.E., Graham C.M. \& Burrows M. 2008: Diversity and evolution of the insect ventral nerve cord. Annu. Rev. Entomol. 53: 253-271.

Park H.H., Park C.W., Kim K.S., Kwon O.S., Han S.S., Hwang J.S., Lee S.M., Seong S.I., Kang S.W., Kim H.R. \& Lee B.H. 2003: Effects of 20-hydroxyecdysone and serotonin on neurite growth and survival rate of antennal lobe neurons in pupal stage of the silk moth Bombyx mori in vitro. Zool. Sci. 20: $111-119$.

Park C.W., Kim J.H., Kim K.M., Hwang J.S., Kang S.W., Kang H.S., Сно B.P., Yu C.H., Kim H.R. \& Lee B.H. 2004: Evidence for brain-derived neurotrophic factor-like neuropeptide in brain of the silk moth Bombyx mori during postembryonic periods. Peptides 25: 1891-1897.

Paschinger K., Rendić D. \& WiLson I.B.H. 2009: Revealing the anti-HRP epitope in Drosophila and Caenorhabditis. Glycocon. J. 26: 385-395.

Prugh J., Croce K.D \& Levine R.B. 1992: Effects of the steroid hormone, 20-hydroxyecdysone, on the growth of neurites by identified insect motoneurons in vitro. Dev. Biol. 154: 331-347.

Rezával C., Werbajh S. \& Cerian M.F. 2007: Neuronal death in Drosophila triggered by GAL4 accumulation. Eur. J. Neurosci. 25: 683-694.

Sakurai S., Kaya M. \& Stake S. 1998: Hemolymph ecdysteroid titer and ecdysteroid-dependent developmental events in the last larval stadium of the silkworm, Bombyx mori: role of low ecdysteroid titer in larval-pupal metamorphosis and a reappraisal of the head critical period. J. Insect Physiol. 44: $867-881$.

SAmbrooK J. \& Russell D.W. 2001: Molecular Cloning: A Laboratory Manual. Vol. 3. CSHL Press, Cold Spring Harbor, NY, pp. A8.40-A8.55.

Sato Y., Shiomi K., Saito H., Imaid K. \& Yamashita O. 1998: Phe-X-Pro-Arg-Leu-NH2 peptide producing cells in the cen- tral nervous system of the silkworm, Bombyx mori. J. Insect Physiol. 44: 333-342.

Schubiger M., Wade A.A., Carney G.E., Truman J.W. \& Bender M. 1998: Drosophila EcR-B ecdysone receptor isoforms are required for larval molting and for neuron remodeling during metamorphosis. Development 125: 2053-2062.

Shannon L.M., Kay E. \& Lew J.Y. 1966: Peroxidase isozymes from horseradish roots. I. Isolation and physical properties. $J$. Biol. Chem. 241: 2166-2172.

Streichert L.C. \& Weeks J.C. 1995: Decreased monosynaptic sensory input to an identified motoneuron is associated with steroid-mediated dendritic regression during metamorphosis in Manduca sexta. J. Neurosci. 15: 1484-1495.

Streichert L.C., Pierce J.T., Nelson J.A. \& Weeks J.C. 1997: Segment-specific programmed cell death of identified motoneurons triggered directly by steroid hormones in vitro. Dev. Biol. 183: 95-107.

Terashima J., Yasuhara N., Iwami M., Sakurai S. \& Sakurai S. 2000: Programmed cell death triggered by insect steroid hormone, 20-hydroxyecdysone, in the anterior silk gland of the silkworm, Bombyx mori. Dev. Genes. Evol. 210: 545-558.

Tettamanti G. \& Malagoli D. 2008: In vitro methods to monitor autophagy in Lepidoptera. Meth. Enzymol. 451: 685-709.

Truman J.W. 1983: Programmed cell death in the nervous system of an adult insect. J. Comp. Neurol. 216: 445-452.

Truman J.W. \& Schwartz L.M. 1984: Steroid regulation of neuronal death in the moth nervous system. J. Neurosci. 4: 274-280.

Truman J.W., Taylor B.J. \& Awad T.A. 1993: The Development of Drosophila melanogaster. Vol. 2. CSHL Press, Cold Spring Harbor, NY, pp. 1245-1275.

WELINDER K.G. 1978: Amino acid sequence studies of horseradish peroxidase. Eur. J. Biochem. 96: 483-502.

Yamanaka A., Tsurumaki J. \& Endo K. 2000: Neuroendocrine regulation of seasonal morph development in a bivoltine race (Daizo) of the silkmoth, Bombyx mori L. J. Insect Physiol. 46: 803-808.

Young C., Klocke B.J., Tenkova T., Choi J., Labruyere J., Qin Y.Q., Holtzman D.M., Roth K.A. \& Olney J.W. 2003: Ethanol-induced neuronal apoptosis in vivo requires BAX in the developing mouse brain. Cell Death Differ. 10: $1148-1155$.

Zee M.C. \& Weeks J.C. 2001: Developmental change in the steroid hormone signal for cell-autonomous, segment-specific programmed cell death of a motoneuron. Dev. Biol. 235: 45-61.

Received April 14, 2009; revised and accepted July 24, 2009 